\title{
MULTI-RESOLUTION MODELING FOR SUPPLY CHAIN SUSTAINABILITY ANALYSIS
}

\author{
Sanjay Jain \\ The George Washington University \\ 2201 G Street, NW, Suite 415 \\ Washington, DC-20052, USA
}

\author{
Sigríður Sigurðardóttir \\ Matis Ltd. \\ Icelandic Food and Biotech R\&D \\ IS-113 Reykjavík, ICELAND
}

\author{
Erik Lindskog \\ Jon Andersson \\ Anders Skoogh \\ Björn Johansson \\ Chalmers University of Technology \\ Product and Production Development \\ SE-41296 Gothenburg, SWEDEN
}

\begin{abstract}
Consumers are increasingly becoming conscious of the need to reduce environmental impact. This has motivated the industry to make efforts to improve the sustainability of their products and supply chains. Such efforts require the ability to analyze the sustainability of supply chains and potential improvements. A systematic approach is needed to evaluate the alternatives that may range from those at the supply chain configuration level to those for improving equipment at a production facility. This paper presents a multi-resolution modeling approach that allows analyzing parts of the supply chain at appropriate level of detail. The capability allows studying the supply chain at high level initially and iteratively drilling down to detailed levels in the identified areas of opportunity and evaluating associated improvement alternatives. Multi-resolution modeling directly relates the impact of improvement in one part of the supply chain to overall supply chain performance thus reducing analyst effort and time.
\end{abstract}

\section{INTRODUCTION}

The level of consciousness regarding the need to reduce the impact of human activity on the environment has been increasing over the recent years. The debate over global warming is not a debate any more for many people. Increasingly volatile weather patterns across the globe have been linked to global warming that in turn has been linked to human activity. The recent natural calamities, in particular the devastation wreaked by Hurricane Sandy on the east coast of the United States, have emphasized the need to reduce environmental impact for many. Many progressive corporations across the globe have started seriously looking for and implementing ways to reduce the environmental impact of their products and the associated supply chains. Yet others have been motivated by the pull from consumers for products with lower impact on environment, that is, increased sustainability than those of the past years.

The task of reducing the environmental impact across supply chains and across life-cycles of products is a challenging one. A prudent approach is to identify the largest opportunities for improving sustainability across a supply chain and address them first. Analyzing the cradle to grave supply chain for such a 
purpose is a daunting task. Previously Jain et al. (2012) proposed a hierarchical approach based on simulation for reducing the environmental impact of supply chains. The approach started with identifying the nodes in a supply chain with the largest opportunities for reducing the environmental impact at a high level using system dynamics simulation. The identified nodes were then modeled at a detailed level using Discrete Event (DE) simulation for further identifying largest opportunities within the nodes for reducing the environmental impact. The use of Agent Based Simulation (ABS) was also proposed at the detailed level for systems involving a large number of independent entities. The applicability of the hierarchical approach was demonstrated using a case study of a supply chain for brakes for industrial forklifts.

The hierarchical approach proposed previously requires developing independent models at successively increasing level of detail in different simulation paradigms as needed. The information discovered using the models with increased level of detail can then be utilized to improve the higher level representations of the supply chain. The successive but independent development of models and iterative updates to models for consistency across levels can be tedious and has the possibility of introducing inconsistencies. In this paper a Multi-Resolution Modeling (MRM) approach for analyzing the sustainability of a supply chain is proposed as an improvement over the hierarchical approach.

The next section presents a literature review of MRM approach and its applications including those for analysis of supply chains. Section 3 describes our implementation of the MRM approach for supply chain sustainability analysis. Section 4 provides a brief overview of a case study that was used previously for demonstrating the hierarchical approach using independent simulation models. The results from the application of MRM approach are discussed in section 5. The results from the application of the hierarchical approach used earlier are compared with those from the MRM approach in section 6. Section 7 concludes the paper with discussion of future research directions.

\section{LITERATURE REVIEW}

Two simulation modeling concepts are specifically relevant to the discussion in this paper: MRM and Hybrid simulation modeling. The two concepts are partly overlapping and share some of the qualities and capabilities. However, the concepts focus on solving different problems. While MRM discusses the use of one or multiple simulation models to analyze systems at varying levels of abstraction, hybrid simulation discusses how to connect and enable different types of modeling techniques to coexist, such as DE simulation and ABS, or DE and System Dynamics (SD). The following two sections briefly review these concepts.

\subsection{Multi-Resolution Modeling Approach}

Jain and Kibira (2010) define MRM as "the capability of executing the complete model or parts of the model at different levels of resolution corresponding to the question being answered with their use." Long term questions may be answered more efficiently with low resolution models. On the other hand, high resolution models may be used for answering near term questions. To help maintain consistency, it will help if short and long term questions can be answered by changing the resolution of the same model. The flexibility to change the resolution of the model will help in other ways too. If the goal is to understand the impact of detailed level decisions in one part of the system on the other parts of the system, the corresponding part of the model that is of interest may be executed at a higher resolution level than others. While the concept can be easily described, there are multiple demanding challenges in MRM identified by Tolk (2006).

MRM may be implemented in multiple ways. The implementation approach can be viewed in two dimensions: modeling paradigm and execution approach. The multiple levels may be represented using the same paradigm, that is, the lowest level of resolution to highest level of resolution are represented using one paradigm such as DE simulation. The other option is to models different level of abstraction using different paradigms, such as, the highest level of abstraction may use SD paradigm, while lower levels of 
abstractions may be represented using DE simulation or ABS. This implementation option can also be viewed as hybrid modeling described in the next section.

On the execution approach dimension, the different resolution level models may be in a single executable file or in multiple executable files. A single executable may be built that includes representations at multiple levels of resolution with the model logic designed to activate different parts of the model as needed by the analysis. Alternatively multiple executable files may be used in a hierarchical scheme with a single executable for the model representing the entire system at the lowest level of resolution and multiple executables used to represent sub-systems at successively higher levels of resolution. Figure 1 shows the matrix of options for implementing MRM.

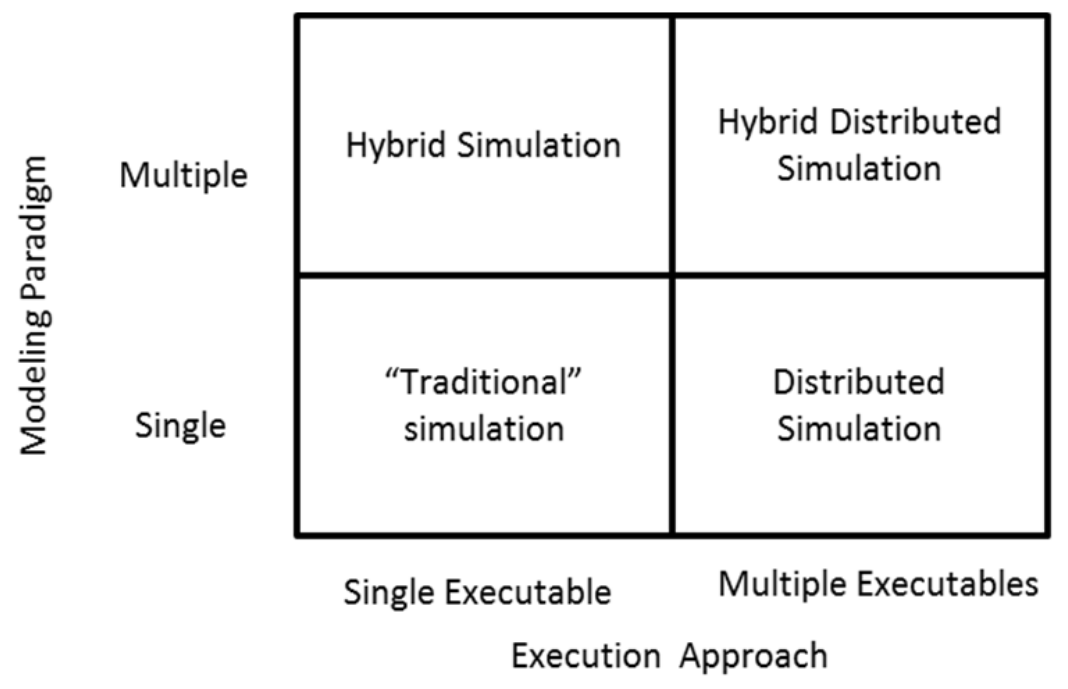

Figure 1: Alternate MRM implementation approaches

Use of multiple executables, whether using the same or different paradigms, requires the use of infrastructure for synchronized execution such as the High Level Architecture. Implementation of such infrastructure is generally quite complex but it allows the benefit of distributed execution on multiple processor machines or on a network of distributed processors that may offer performance advantage. Such performance comparison is not within the scope of this paper.

A few of the recent software allow including models of different paradigms in the same executable and thus eliminate the complexity of implementing mechanisms for synchronized execution. A subset of such software may exploit multi-threaded execution on multi-processor machine and thus offer performance advantage without the complexity of mechanisms for synchronized execution.

MRM implies synchronized execution of the multiple levels of abstraction. Successive executions of models at different levels of execution such as those utilized in Jain et al. (2012) are generally not identified as MRM.

MRM approaches have started to appear in multiple application areas including climate (Ringler 2011), train operations (Jiang, Ji, and Liu 2011), logistics system (Wu, Qi, and Liu 2011), factory system (Shao et al. 2012), emergency evacuation (Yang, Wu, and Ren 2012), and vehicle infrastructure (Li, Wei, and Cai 2012). Hong and Kim (2013) define a specification for describing the MRM space and present an example of an attack by a group of airplanes on a target to demonstrate its use.

\subsection{Hybrid Simulation Modeling}

Hybrid Simulation Modeling enables the integration of different modeling techniques to solve a specific question. Lättiläa, Hilletofthb, and Linc (2010) described the concept using examples with ABS and SD 
modeling, and presented five approaches to combine these techniques. Tako and Robinson (2012) used hybrid simulation modeling combining DE and SD. Furthermore, Rabelo et al. (2005) used a hybrid SD and DE model to simulate a production system at an enterprise level. They argue that DE simulation itself is not suitable for high level perspective but can be successfully combined with a SD approach. Heath et al. (2011) compared and discussed different hybrid solutions including ABS-DE, ABS-SD, and DE-SD. They state that hybrid simulation modeling holds substantial potential but found out that the software support is still insufficient despite the recent major advances.

\subsection{Simulation for Sustainability Analysis}

Manufacturing simulations combined with sustainability analyses have been developed and applied in research for a decade (Reinhard et al. 2011; Jimenez et al. 2013; Wohlgemuth et al. 2004; Heilala et al. 2008). A driving force for such combined approach is to avoid analysis and experiments in running production but still provide the production engineers with a capability to analyze the environmental impact of a production system. The simulation-based approach enables accurate analysis for both current and future system states. However, the industrial dissemination is lagging behind. Possible reasons are the vast amounts of data required (Jahangirian et al. 2013) and difficulties in finding the appropriate resolution level for the simulation model and related analysis.

A majority of the published applications establish a clear focus on reducing energy consumption in selected parts of a supply chain instead of taking a holistic environmental impact perspective. An application focusing on impact measures for parts of a system rather than a holistic impact perspective requires shorter time for analysis but such analysis could potentially miss important aspects of the environmental impact. Recently, there is a trend towards using more holistic measures such as carbon footprint and other similar environmental impact indicators. The use of such holistic measures reduces the risk for suboptimization and improves the accuracy of analyses, but it also increases the amount of data needed. An example of use of holistic measures is provided by Laurent, Olsen, and Hauschild (2011) who use carbon footprint as an indicator of sustainability performance in manufacturing industry. This standpoint aligns well with human health issues and is easy to communicate to customers and other actors in society. A recent review by Mani et al. (2013) proposed to use energy, CO2 equivalents, waste, water and a couple of other defined emissions as key performance indicators in manufacturing industry. A general suggestion is to stick to a few holistic indicators to enable faster analysis and limit the data requirements.

\section{MRM IMPLEMENTATION}

The MRM implementation reported in this paper is based on the approach utilizing multiple paradigms with a single executable file. The use of multiple paradigms also qualifies the implementation to be identified as hybrid simulation. A supply chain is represented at the lowest level of resolution using a SD model. It is proposed that the DE simulation models be used to represent the operations at each node of the supply chain at the next higher level of resolution. This will enable a dynamic analysis of the supply chain at a low resolution level as well as at a detailed level. Any node(s) of special interest in the supply chain can be analyzed at a more detailed level while the rest of the supply chain is modeled at a low resolution level. For the high resolution level modeling of a node it will be possible to analyze specific phases, such as production of components, in the very detailed way enabled by the DE simulation paradigm. Changes on that level will directly affect the system level model and other nodes without any need to update the input to the SD model. Changes at the SD level, such as production demand, will also be communicated directly to the nodes modeled at the high resolution level using DE simulation. The aim with this approach is to have a dynamic simulation model of the supply chain that creates a good overall picture of the system and at the same time allows the flexibility to delve deeper for the nodes of interest. 


\section{CASE DESCRIPTION}

To demonstrate the proposed MRM implementation presented in section 3, a cradle-to-cradle supply chain system for a forklift brake-set lifecycle is used. The data originates from the case presented in Lindskog et al. (2011) and Jain et al. (2012) with some minor adjustments to fit the MRM approach. The supply chain system is analyzed for its carbon footprint and associated tradeoffs. The cradle-to-cradle phenomenon is represented by the closed-loop supply chain starting from steel production, running through manufacturing and use phase, and ending with recycling of used brakes as raw material at the steel plant. Figure 2 shows a representation of a general supply chain in the context of a forklift brake lifecycle. For this initial demonstration of the implementation only the brake manufacturer node is modeled at the high resolution with DE while the supply chain is modeled at a low resolution using SD. The successive nodes in the modeled supply chain are listed below:

i. Iron ore mine

ii. Steel plant

iii. Brake component suppliers

iv. Brake manufacturer

v. Forklift manufacturer

vi. Industrial user of forklifts

vii. Disassembler (recyclable steel components sent back to steel plant as raw materials to reduce the need for mined iron ore)

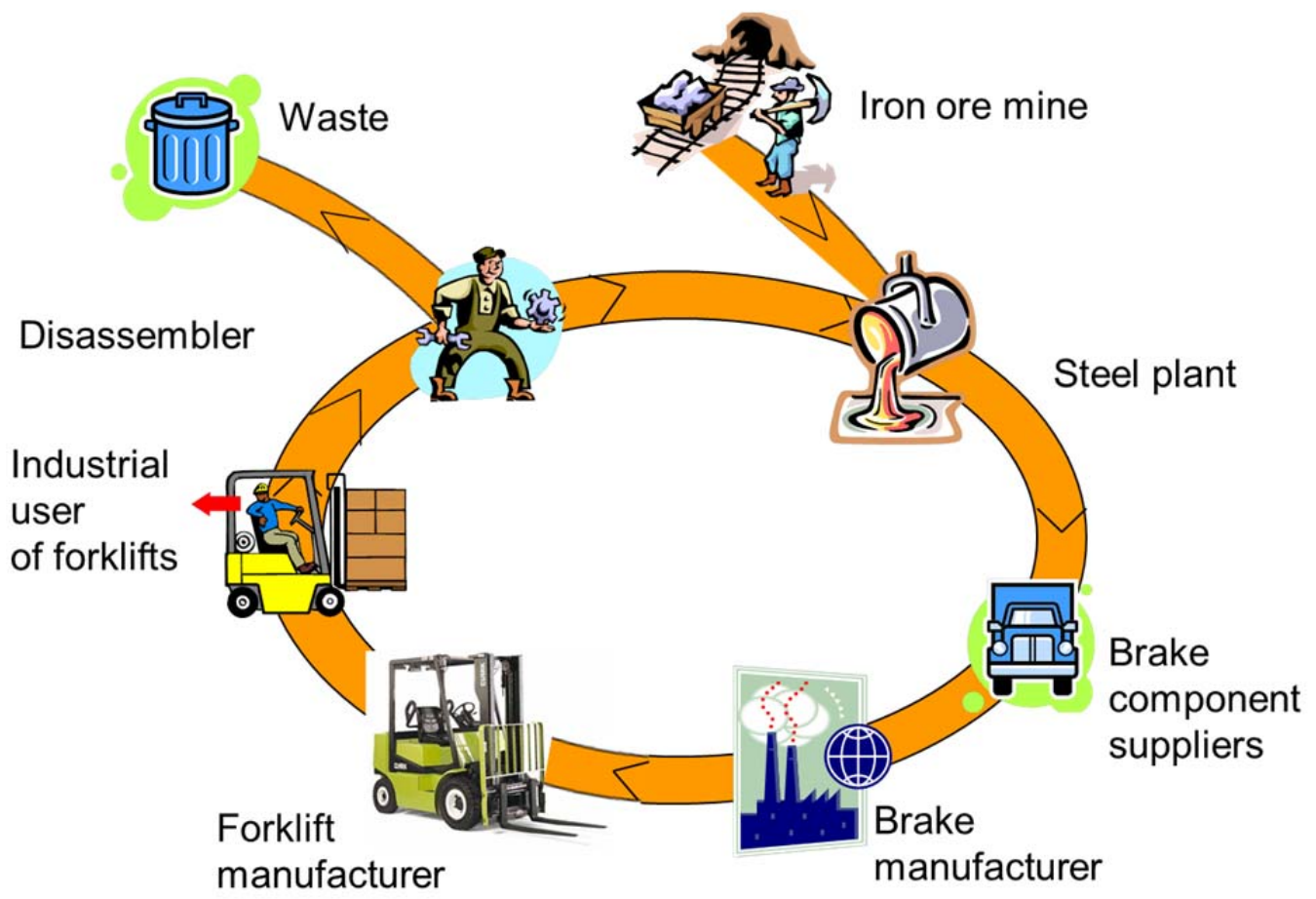

Figure 2: Life cycle of a brake-set

This supply chain system was modeled using the MRM approach with hybrid SD and DE models. In the following sub-sections the models at different levels of resolution are discussed first followed by the interactions among the supply chain SD model and the production node DE model. 
Jain, Sigurðardóttir, Lindskog, Andersson, Skoogh, and Johansson

\subsection{Low Resolution Supply Chain Model}

The entire supply chain was represented at the lowest level of resolution (or at the highest level of abstraction) using the SD approach. The model captures the supply chain for the entire lifecycle of a forklift brake-set, and is quite parallel to the SD model described in Jain et al. (2012). For the MRM implementation the earlier implementation of the supply chain SD model was imported into a software that allows hybrid modeling, that is, allows creating models using different simulation paradigms including SD, DE, and ABS. The new implementation of the supply chain SD model was validated against the results from the implementation of the corresponding SD model reported in Jain et al. (2012).

Some obstacles had to be overcome when importing the model implemented using one software into the other. For instance, a number of mathematical expressions did not translate correctly and moreover, problems were discovered with some of the conditional flows from one stock to another. This was solved for the flows with the discovered problems by adding functions with Java code that were called in each time step.

\subsection{High Resolution Brake-set Manufacturer Node Model}

The brake-set manufacturer node of the supply chain was modeled using the DE simulation paradigm to demonstrate the proposed approach. The brake-set manufacturer node was selected for the high resolution model because this was the node in the supply chain where the corresponding industry partner allowed access to more detailed data. Ideally, the node that offers the most opportunity for meeting the goals, in this case carbon footprint reduction, should be the one that should be modeled at higher resolutions.

The brake-sets consist of a number of different components assembled into four products of two types. The products mainly consist of metal components machined at the factory from raw steel sheets and tubes and some pre-made components. In the model the logic was designed to determine carbon drivers for each component as it was processed by a resource and for all the components as they were assembled together to produce the brake-set. This design made it possible to continuously track the carbon drivers during the simulation run for each product or component individually as well as for the total production. The overhead carbon drivers, such as energy for heating, lights, and cooling liquids, were allocated to the product at the end of the process. The overhead contribution to the product was calculated from an overhead percentage based on the product's weight compared with the total production by weight at the facility. Power quality monitoring instruments were used to analyze the energy consumption for individual machines for use in the model. Energy consumption was measured for idle and busy states of the individual machines and for uses classified as overhead such as heating and cooling of the facility. Other carbon drivers were estimated based on data on production, invoices, and life cycle inventory databases.

The brake-set manufacturer operations were previously modeled using a DE software with an advanced 3D visualization capability. An import facility was not available from that software to the software used for the hybrid simulation reported in this study. The DE simulation model hence had to be reimplemented. This provided an opportunity to set up the model specifically for integration in the MRM structure. The real manufacturing site is organized around stand-alone resources with no structured material flow. The components are transported between the resources according to individual work process schedules. This complex flow was modeled in detail within the previous model. A simplified structure was used for the implementation in the hybrid model commensurate with the goals of this study. The main difference between the two models is that in the hybrid model all resources are organized in one straight workflow. Resource specific values, such as energy, operation, and setup times, were recalculated to fit the new simplified structure.

\subsection{Interaction between Models at Different Resolutions}

The low resolution supply chain SD model directly linked to the high resolution DE model of the brakeset manufacturer operations. The logic has been set up to allow use of either the low resolution or the high 
resolution model of the brake-set manufacturer node. The initial idea was to create the following connections between the two models:

SD to DE:

- Brake-set order quantity for the month for the brake-set manufacturer based on the inventory position of brake-sets at the forklift assembler

- Produced brake-set components arriving at the brake-set manufacturer

DE to SD:

- Brake-sets produced for the month

- Order quantity for the month for the components based on the inventory position at the brake-set manufacturer

- Energy used in brake-set production per month

- Emissions due to brake-set production per month

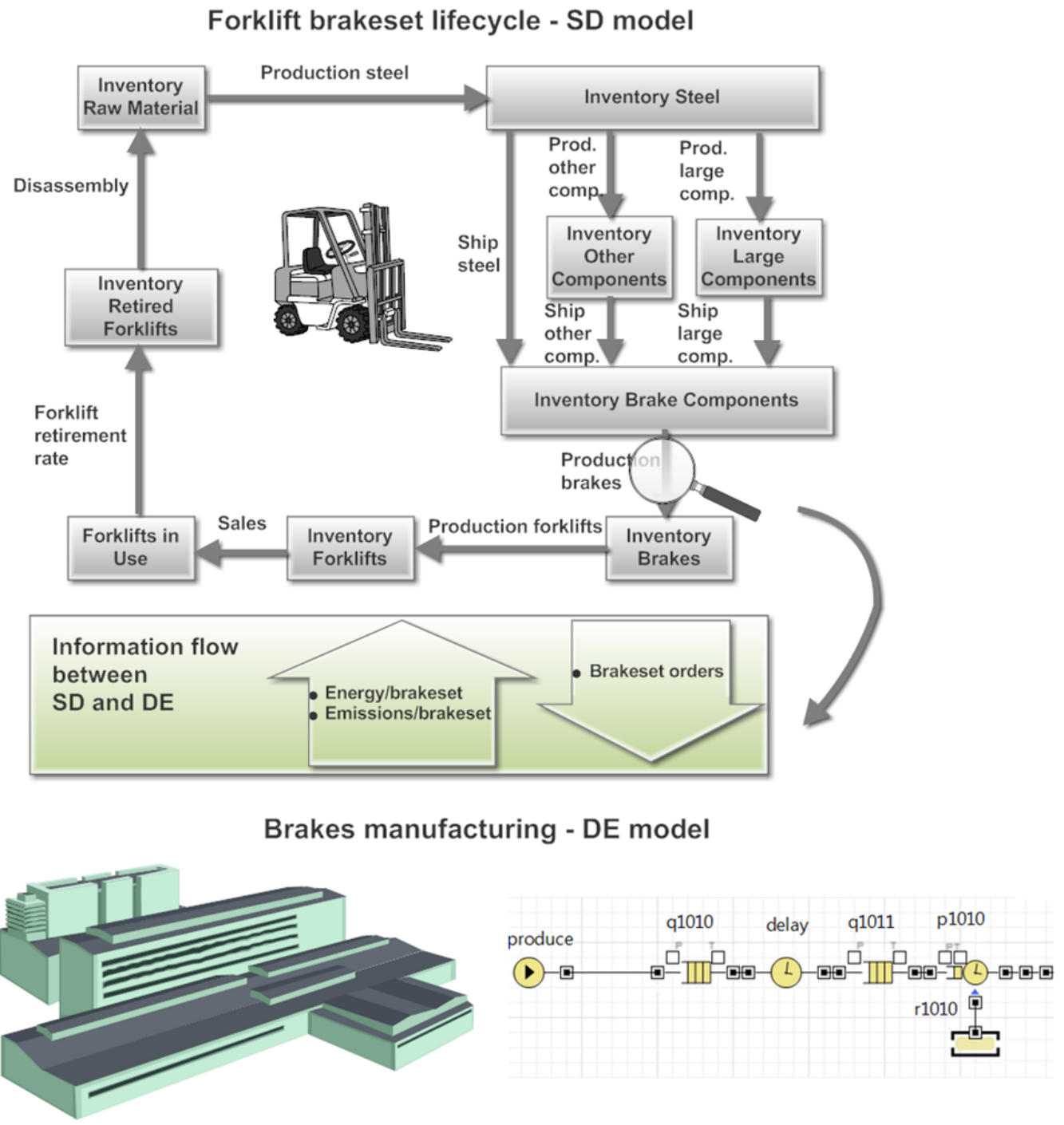

Figure 3: Interactions between the low resolution SD model and high resolution DE model. 
In this particular case study, the original DE model was simplified excluding many of the complicated logics in the manufacturing process. For that reason only brake-set order quantity was passed from the SD model to the DE model and the emissions and the energy per month from the DE model to the SD model. Figure 3 shows a diagram of the SD model and how it interacts with the DE model.

\section{RESULTS AND ANALYSIS}

The process of creating a hybrid model that made it possible to run both SD and DE models simultaneously and pass information between the two required some special attention to details as shifting from two different modeling software to another was not a straightforward task. Different modeling platforms call for different coding skills. When verifying the model against previous results there were differences in the intermediate calculations while total and final results are quite close. Table 1 shows a comparison of total results from the different models of carbon footprint throughout the whole life cycle of a brakeset. These results should be observed for the purpose of comparing the models only, and regarded as estimates and not highly accurate results for the actual carbon footprint of brake-sets.

Table 1: Results of total carbon footprint for the life cycle of brake-sets for different models, obtained from simulation runs over 120 months.

\begin{tabular}{l|c} 
& $\begin{array}{c}\text { Total carbon footprint } \\
{\left[\mathrm{KgSCO}_{2} \text { eq }\right]}\end{array}$ \\
\hline Jain et al. (2012) & $3,012,555$ \\
New SD model only & $3,105,713$ \\
SD-DE Hybrid model & $3,091,329$
\end{tabular}

Figure 4 shows results for the total carbon footprint in the whole life cycle of the brake-sets. The blue line shows results from the model created by Jain et al. (2012), the red line results from our new SD model and the green line shows results for the SD-DE hybrid model.

\section{COMPARISON OF APPROACHES}

The approach described in this paper is different compared with the one used in Jain et al. (2012). In the previous approach two different models were used; one DE model to simulate the factory level of brakeset manufacturer and a SD model to represent the overall supply chain. Compared with the approach presented in this paper the previous models were isolated from each other and values were manually transferred using only average values between two different computers to update the data. In the currently presented approach the complete translation of data is performed within the same model environment and dynamics are hence more accurately modeled. Jovanovski et al. (2012) describe the same approach, with a very brief and fictive case description on how the SD and DE parts of the model interact. This paper discusses the combination further as well as describes a real case example and draws conclusions from that reasoning and experience.

Brailsford, Desai and Viana (2010) made a similar comparison on two healthcare cases, they found that the "big picture" provided by the SD model is worth the modeling work to a certain point. In their examples the models could have been built completely using only the DE functionality, but it would have been a very cumbersome and time-consuming process. They conclude with asking themselves if the research progress is closer to combining SD and DE since year 2000, and answering that it was only to a certain point, not completely. The approach shown in this paper is an integrated model in one single software and hence, the combination may be considered complete. 
Jain, Sigurðardóttir, Lindskog, Andersson, Skoogh, and Johansson

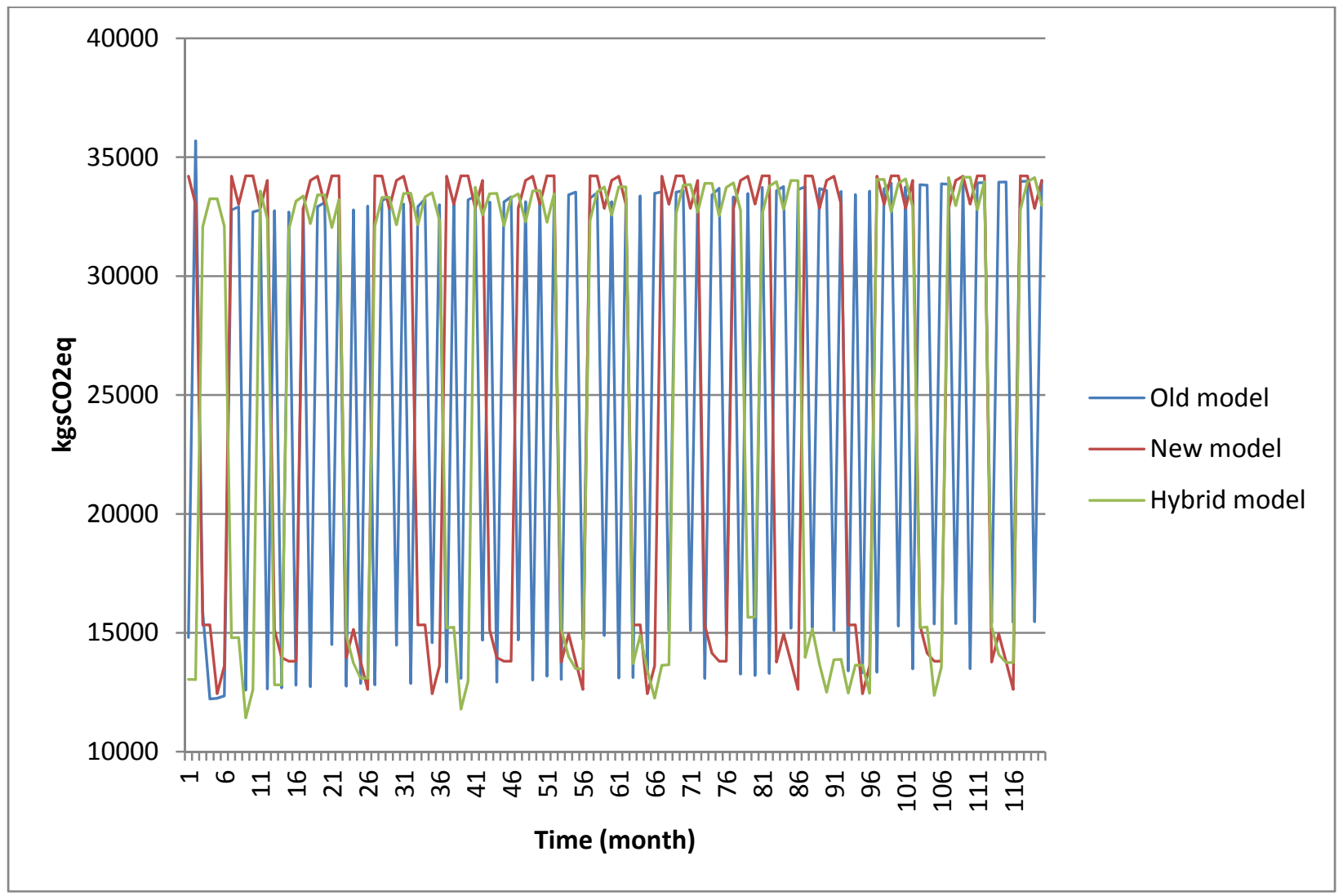

Figure 4: Total carbon footprint across the supply chain as reported by different models

Some discussion on the pros and cons is still valid since the modeling work is not yet that user friendly. MRM requires more skill and takes longer time to complete than just creating either the SD or the DE model separately. The modeling approach used as test for the reasoning in this paper was cumbersome and gave new experiences, such as:

- The SD and DE environments are still not smoothly integrated, even though they are modeled in the same environment. This is due to the fact that the SD part of the model used time-steps which needs to be followed by the DE part of the model to update the overall SD model in a proper manner. This limits the interaction and real world replication. The total runtime for the combined model is limited to follow this pattern as well.

- Stochastic machine downtimes are modeled in the combined model, but information on such occurrences were not communicated between the separated models in the earlier approach.

- It is not apparent and easy to determine which step length to use, since different steps can be preferred by different nodes.

However if the MRM is done wisely, i.e., using DE for the most interesting part of a system, which normally is the part which limits system performance most, the authors believe that it is useful and aids decision-makers to pinpoint the problems more accurately than using only SD or only DE models.

\section{CONCLUSION}

The contribution of this paper is to show the value of MRM for sustainability analysis of supply chains. MRM applications have started appearing for several different domains, however, few can be found in the 
area of supply chain sustainability. The need for analysis at different level of resolution within the supply chain has been recognized previously but the few prior attempts used separate models such as those used in Jain et al. (2012). The MRM application facilitates the sustainability analysis of supply chain by allowing to address key nodes of the supply chain at higher resolution while rest of the supply chain is modeled at low resolution. The MRM approach enables an efficient utilization of the expensive and highly skilled modeling resources by focusing the effort for high resolution modeling where necessary and eliminating the effort for manually transferring data between models. MRM approach has been further facilitated by recent availability of software that allow modeling using different paradigms within a single executable thus eliminating the need for implementing complex mechanisms for distributed simulation. On the other hand, MRM requires more advanced modeling skills than for traditional models particularly in the implementation using different modeling paradigms.

Furthermore, the article presents an approach to classify multi-resolution models using the two dimensions of modeling paradigm and execution approach and identified the overlap between MRM and hybrid modeling. The field is evolving and requires a corresponding evolution of the terminology for unambiguously describing the new concepts. The authors hope the discussion points to the need for standardization of definitions of MRM and hybrid modeling.

Future work is expected to continue expanding MRM application for supply chain sustainability analysis in particular for the issues that are complicated by interactions of a number of human players. Addressing such issues may require employing ABS paradigm in addition to the combination of SD and DE paradigms reported here.

\section{ACKNOWLEDGMENTS}

The work of Sigríður Sigurðardóttir within this research was funded by Matís - Icelandic Food and Biotech R\&D, that support is gratefully acknowledged.

The work of Erik Lindskog, Jon Andersson, Anders Skoogh, and Björn Johansson on this research was funded by a grant from ProViking through the EcoProIT project and by VINNOVA (Swedish Agency for Innovation Systems) through the NFFP5 program. This work has been carried out within the Sustainable Production Initiative and the Production Area of Advance at Chalmers University of Technology, which is gratefully acknowledged.

\section{REFERENCES}

Brailsford, S.C., S.M. Desai and J. Viana. 2010. "Towards the Holy Grail: Combining System Dynamics and Discrete-Event Simulation in Healthcare." In: 2010 Winter Simulation Conference, edited by B. Johansson, S. Jain, J. Montoya-Torres, J. Hugan, and E. Yücesan, 2293-2303. Piscataway, NJ: IEEE, Inc.

Heath, S.K., A. Buss, S.C. Brailsford, and C.M. Macal. 2011. "Cross-paradigm Simulation Modeling: Challenges and Successes." In: Proceedings of the 2011 Winter Simulation Conference, edited by S. Jain, R. R. Creasey, J. Himmelspach, K. P. White, and M. Fu, 2788-2802. Piscataway, NJ: IEEE, Inc.

Heilala, J., V. Saija, H. Tonteri, J. Montonen, B. Johansson, J. Stahre, and S. Lind. 2008. "SimulationBased Sustainable Manufacturing System Design." In: Proceedings of the 2008 Winter Simulation Conference, edited by S.J. Mason, R.R. Hill, L. Mönch, O. Rose, T. Jefferson, J.W. Fowler, 19221930. Piscataway, NJ: IEEE, Inc.

Hong, S.-Y., and T. G. Kim. 2013. "Specification of Multi-Resolution Modeling Space for Multiresolution System Simulation." Simulation 89(1): 28-40.

Jain, S., E. Lindskog, and B. Johansson. 2012. "Supply Chain Carbon Footprint Tradeoffs Using Simulation." In: Proceedings of the 2012 Winter Simulation Conference, edited by C. Laroque, J. Himmelspach, R. Pasupathy, O. Rose, and A.M. Uhrmacher. Piscataway, NJ: IEEE, Inc. 
Jain, S., and D. Kibira. 2010. "A Framework for Multi-Resolution Modeling of Sustainable Manufacturing." In: 2010 Winter Simulation Conference, edited by B. Johansson, S. Jain, J. Montoya-Torres, J. Hugan, and E. Yücesan, 3423-3434. Piscataway, NJ: IEEE, Inc.

Jahangirian, M., T. Eldabi, A. Naseer, L. K. Stergioulas and T. Young. 2010. "Simulation in Manufacturing and Business: A Review." European Journal of Operational Research 203:1-13.

Jiang, X., X. Ji and J. Liu. 2011. "Multi-Resolution Modeling of High Speed Railway Infrastructure for Train Operation Simulation." Applied Mechanics and Materials 97-98:560-565.

Jimenez, E., E. Martinez, J. Blanco, M. Perez, and C. Graciano. 2013. "Methodological Approach Towards Sustainability by Integration of Environmental Impact in Production System Models Through Life Cycle Analysis: Application to the Rioja Wine Sector." Simulation, Prepublished March 15, 2013, doi:10.1177/0037549712464409.

Jovanovski, B., R. Minovski, S, Voessner and G. Lichtenegger. 2012. "Combining System Dynamics and Discrete Event Simulations - Overview of Hybrid Simulation Models." Journal of Applied Engineering Science 10(3):135-142.

Laurent, A., S.I. Olsen, and M.Z. Hauschild. 2010. "Carbon Footprint as Environmental Performance Indicator for the Manufacturing Industry." CIRP Annals - Manufacturing Technology 59(1):37-40.

Li, S.-H., S. Wei, and B.-G. Cai. 2012. "Infrastructure Research and Multi-resolution Modeling of Cooperative Vehicle-infrastructure System." In: CICTP 2012@ Multimodal Transportation SystemsConvenient, Safe, Cost-Effective, Efficient, 1515-1526. ASCE.

Lindskog, E., L. Lundh, J. Berglund, T. Lee, A. Skoogh, and B. Johansson. 2011. "A Method for Determining the Environmental Footprint of Industrial Products using Simulation." In: Proceedings of the 2011 Winter Simulation Conference, edited by S. Jain, R.R. Creasey, J. Himmelspach, K.P. White, and M. Fu, 2136-2147. Piscataway, NJ: IEEE, Inc.

Lättilä, L., P. Hilletofth, and B. Lin. 2010. "Hybrid simulation models - When, Why, How?" Expert Systems with Applications 37:12 7969-75.

Mani, M., J.H. Lee, K.W. Lyons, and S.K. Gupta. 2013. Review on Sustainability Characterization for Manufacturing Processes. NISTIR-7913. National Institute of Standards and Technology, USA.

Rabelo, L., M. Helal, A. Jones, and H.-S.Min, 2005. "Enterprise Simulation: A Hybrid System Approach." International Journal of Computer Integrated Manufacturing 18(6):498-508.

Reinhard, J., M.F. Emmenegger, A.H. Widok, and V. Wohlgemuth. 2011. "RSB Tool: A LCA Tool for the Assessment of Biofuels Sustainability." In: Proceedings of the 2011 Winter Simulation Conference, edited by S. Jain, R.R. Creasey, J. Himmelspach, K.P. White, and M. Fu, 1048-59. Piscataway, NJ: IEEE, Inc.

Ringler, T. 2011. "Approaching the Problem of Regional Climate Simulation through a Global, MultiResolution Modeling Approach: Successes and Challenges." In: AGU Fall Meeting Abstracts, 1:4.

Shao, X.Y., Z.L. Guan, C.J. Wang, and Y.F. Wu. 2012. "A Framework of Digital Factory System Using Multi-Resolution Simulation." Applied Mechanics and Materials 159: 12-17.

Tako, A.A., and S. Robinson. 2012. "The Application of Discrete Event Simulation and System Dynamics in the Logistics and Supply Chain Context." Decision Support Systems 52(4):802-15.

Tolk, A. 2006. "Multi-Resolution Challenges for Command and Control M\&S Services." In: Proceedings of the 2006 Spring Simulation Interoperability Workshop, Simulation Interoperability and Standards Organization (SISO), Paper Number 06S-SIW-007.

Yang, B., Y.-G. Wu, and B. Ren. 2012. "Application of Multi-Resolution Modelling in Emergency Evacuation Simulation." International Journal of Simulation and Process Modelling 7(1): 89-97.

Wohlgemuth, V., B. Page, M. Mäusbacher, and P. Staudt-Fischbach. 2004. "Component-Based Integration of Discrete Event Simulation and Material Flow Analysis for Industrial Environmental Protection: A Case Study in Wafer Production." In: Proceedings of the 18th International Conference for Environmental Protection, October 21-23, CERN, Geneva, p. 303-312. 
Wu, Y., E. Qi, and L. Liu. 2011 “A Research on Express Logistics System Simulation Based on MultiResolution Modeling." In: International Conference on Management and Service Science (MASS), 15. IEEE.

\section{AUTHOR BIOGRAPHIES}

SANJAY JAIN is an Associate Professor in the Department of Decision Sciences, School of Business at the George Washington University. Before moving to academia, he accumulated over a dozen years of industrial R\&D and consulting experience working at Accenture in Reston, VA, USA, Singapore Institute of Manufacturing Technology, Singapore and General Motors North American Operations Technical Center in Warren, MI, USA. He is an associate editor of the International Journal of Simulation and Process Modelling and a member of the editorial board of the International Journal of Industrial Engineering. His research interests are in application of modeling and simulation to complex scenarios including sustainable supply chains and project management. His email address is jain@email.gwu.edu.

SIGRÍĐUR SIGURĐARDÓTTIR is a PhD candidate in Industrial Engineering at the University of Iceland working on a research project in collaboration with Matis ltd.- Icelandic Food and Biotech R\&D. She graduated with a Master's degree in 2011 from the same institute and spent a year at Chalmers University of Technology in Gothenburg in Sweden during her studies. In fall 2011 she was a visiting scholar at the IEOR department at UC Berkeley. Her email address is sigridursig@matis.is.

ERIK LINDSKOG is a PhD student in the field of virtual production systems at the Department of Product and Production Development, Chalmers University of Technology, Sweden. In 2011 he obtained his M.Sc. degree in Automation and Mechatronics from the same university. Erik is researching mainly on how to visualize and convey information for sustainability considerations while designing and operating manufacturing systems. His email address is erik.lindskog@chalmers.se.

JON ANDERSSON is a PhD student in the field of virtual production system at the Department of Product and Production Development, Chalmers University of Technology. He is currently active in the field of simulation of production flows and especially in a project called EcoProIT (http://www.ecoproit.com). The project aims to develop methodologies and tools for environmental impact assessment in DE simulation. His email address is jon.andersson@chalmers.se.

ANDERS SKOOGH is an Assistant Professor in the area of Virtual Production at the Department of Product and Production Development, Chalmers University of Technology, Sweden. In 2011, he received his $\mathrm{PhD}$ in Production Systems from Chalmers. Before starting his research career, he accumulated industrial experience as a logistics developer at Volvo Car Corporation. His main research area is virtual tools for decision support in development of sustainable production systems. The current focus is on efficient management of production data, mainly for simulation and maintenance purposes. Anders is also the Director of Chalmers' Masters Programme in Production Engineering. His email address is anders.skoogh@chalmers.se.

BJÖRN JOHANSSON is an Associate Professor in the field of virtual production system and Vice Head of Production Systems division at the Department of Product and Production Development, Chalmers University of Technology, Sweden. He serves as Production Modeling Corporation director for the European office in Gothenburg. His main research interest is in the area of Sustainable Production with a multidisciplinary approach including sustainability coaching, on social, environmental and economic analysis, Discrete Event Simulation applied for manufacturing industries, environmental effects modeling, and visualization of sustainability metrics. His email address is bjorn.johansson@chalmers.se. 